\title{
ON HOMEOMORPHISM SPACES OF HILBERT MANIFOLDS
}

\author{
RAYMOND Y. WONG
}

\begin{abstract}
Let $M$ be a Hilbert manifold modeled on the separable Hilbert space $l_{2}$. We prove the following Fibred Homeomorphism Extension Theorem: Let $M \times B^{n}$ $\rightarrow B^{n}$ be the product bundle over the $n$-ball $B^{n}$, then any (fibred) homeomorphism on $M \times S^{n-1}$ extends to a homeomorphism on all $M \times B^{n}$ if and only if it extends to a mapping on all $M \times B^{n}$. Moreover, the size of the extension may be restricted by any open cover on $M \times B^{n}$. The result is then applied to study the space of homeomorphisms $\mathcal{H}(M)$ under various topologies given on $\mathcal{H}(M)$. For instance, if $M=l_{2}$ and $\mathcal{H}\left(l_{2}\right)$ has the compact-open topology, the $\mathcal{H}\left(l_{2}\right)$ is an absolute extensor for all metric spaces. A counterexample is provided to show that the statement above may not be generalized to arbitrary manifold $M$.
\end{abstract}

1. Let $\mathcal{H}(M)$ be the space of homeomorphisms of an $n$-manifold $M$ with the c-o (compact-open) topology and let $\mathcal{H}_{\partial}(M)$ be the subspace that is the identity on $\partial M$ (the boundary of $M$ ). If $M$ is compact, $\mathcal{H}(M)$ is locally contractible [Ce, Ed-Ki] and for $n \leqslant 2, \mathcal{H}(M)$ is an ANR (Absolute neighborhood retract) [An, Lu-Ma]. The main problem as yet unsettled is whether $\mathcal{H}_{\partial}(M)$ are ANR's for $n \geqslant 3$. If the answer turns out to be affirmative, then they are Hilbert manifolds [Ge, To].

If $M$ is noncompact, $\mathcal{H}(M)$ is locally contractible when supplied with the majorant topology (see §2) [Ce]. We consider here the space of homeomorphisms $\mathcal{H}(M)$ of a separable infinite dimensional space $M$ and equip $\mathcal{H}(M)$ with either the c-o or majorant topology. If $M$ is the Hilbert cube $Q=[-1,1]^{\infty}, \mathcal{H}(Q)$ is known to be contractible [Re, Wo]. In general $\mathcal{H}(M)$ is locally contractible when $M$ is a compact $Q$-manifold [Ch]. A remarkable result of Ferry [Fe] and Toruńczyk [To] showed that $\mathcal{H}(M)$ is, in fact, an ANR.

Let $M$ be a separable Hilbert manifold modeled on the Hilbert space $l_{2}$. The function space, $e(M)$, can be equipped with the c-o or majorant topology. Here the topology plays an important role since $\mathcal{H}(M)$ may fail to be locally contractible in the c-o topology. The following example illustrates that point.

EXAMPLE 1. We remove from the open interval $(0,1)$ a sequence of disjoint open intervals which have their diameters tend to zero as they approach 1 . Denote the resulting space by $X$. Let $Y$ denote the space obtained by attaching circles to the end points in $X$ as illustrated in the figure below. According to [We], $M=Y \times l_{2}$ is a Hilbert manifold. But, by switching the upper and lower half of smaller and smaller

Received by the editors October 16, 1981 and, in revised form, November 18, 1982.

1980 Mathematics Subject Classification. Primary 58D15, 58D05; Secondary 54C55.

Key words and phrases. Hilbert manifolds, spaces of homeomorphisms, absolute neighborhood extensors, Z-sets. 
circles, we can obtain homeomorphisms which are contained in any neighborhood of the identity $e$ (on $M$ ) under the c-o topology yet clearly can not be deformed to $e$.

The purpose of this paper is to investigate the homeomorphism spaces of Hilbert manifold $M$ under different topologies. Our main tool is the fibred homeomorphism extension theorem (Theorem 1) and our main applications and examples are contained in §3. The major effort of this paper is to give a proof of Theorem 1 , and we will do that in $\S \S 4$ and 5 .

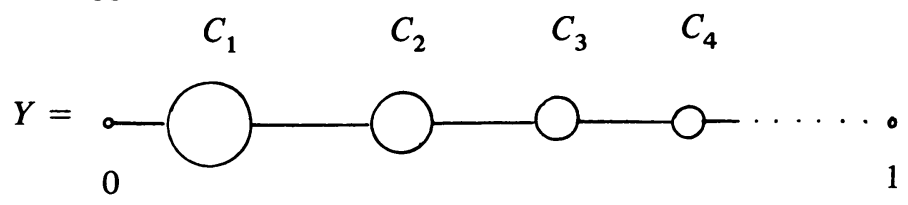

FIGURE 1

We are indebted to the valuable observations and comments by T. Dobrowolski, who contributes greatly in the final write up of $\S 3$.

2. Statement of main theorem. Let $M$ denote a separable, complete, metric Hilbert manifold. Let $\left(M \times B^{n}, p, B^{n}\right)$ be the product bundle over the base space $B^{n}$ ( $n$-ball). Denote the projection $M \times B^{n} \rightarrow M$ by $q$. Let $\operatorname{Mesh}(A)$ denote the (metric) diameter of a subset $A$ in a metric space $X$. Here is our Fibred Homeomorphism Extension Theorem; the proof will be given in $\$ \S 4$ and 5 . (See $\$ 4$ for further definition and notation.)

TheOREM 1. Suppose, for $n \geqslant 1, h: M \times B^{n} \rightarrow M \times B^{n}$ is a fibred map such that $h \mid M \times S^{n-1}$ is a homeomorphism onto $M \times S^{n-1}$. Then for any fixed $k>0$, there is a fibred homeomorphism $h^{\prime}: M \times B^{n} \rightarrow M \times B^{n}$ extending $h \mid M \times S^{n-1}$ such that for any $m \in M, q h^{\prime}\left(\{m\} \times B^{n}\right) \subset N(m)$, where $N(m)$ is the $\varepsilon(m)$-neighborhood of $q h\left(\{m\} \times B^{n}\right)$ in $M$ with $\varepsilon(m)=\operatorname{Mesh}\left(q h\left(\{m\} \times B^{n}\right)\right) / k$.

REMARK. It is important to observe that if

$$
\operatorname{Mesh}\left(q h\left(\{m\} \times B^{n}\right)\right)=0,
$$

$q h\left(\{m\} \times B^{n}\right)$ is a point $\{p\}$. The lemma requires that $q h^{\prime}\left(\{m\} \times B^{n}\right)=\{p\}$.

3. Application to homeomorphism spaces. Throughout the following, $M$ will denote a separable, complete, metric Hilbert manifold modeled on the Hilbert space $l_{2}$ of all square summable real sequences. In fact (by [He]) we assume that $M$ is an open set in $l_{2}$.

The majorant topology on the mapping space $e(X, Y)$ is the topology where for any $f \in \mathcal{C}(X, Y)$, a fundamental neighborhood system of $f$ consists of $\{N(f, \mathcal{Q})\}$, where $\mathscr{U}$ is any open cover of $Y$ and

$$
N(f, \mathscr{U})=\{g \in \mathcal{C}(X, Y): g \text { is } \text { U-close to } f\} \text {. }
$$

$g$ is U-close to $f$ means that for every $x \in X,\{f(x), g(x)\} \in U$ for some $u \in \mathcal{U}$. Alternatively, the majorant topology on metric spaces can also be defined as follows: For any $f \in \mathcal{C}(X, Y)$, a fundamental neighborhood system of $f$ consists of $\left\{N_{\delta}(f)\right\}$, where $\delta: Y \rightarrow(0, \infty)$ is any map and

$$
N_{\delta}(f)=\{g \in \mathcal{C}(X, Y): d(f(x), g(x))<\delta(f(x)) \text { for all } x \in X\} .
$$


We use $B^{n}, S^{n}$ to denote respectively the $n$-ball and $n$-sphere.

Let $\mathcal{H}_{\mathrm{c}-\mathrm{o}}(M)$ and $\mathcal{H}_{m}(M)$ denote respectively the homeomorphism spaces of $M$ onto $M$ under the compact-open (c-o) topology and the majorant topology.

A space $Y$ is called an Absolute Extensor for metric spaces (respectively Absolute Neighborhood Extensor), denoted $Y \in \operatorname{AE}(\Re)$ ) (resp. $Y \in \operatorname{ANE}(\Re)$ ) if, given any closed set $A$ in a metric space $X$ and any map $f: A \rightarrow Y$, there is a map $\tilde{f}$ of $X$ into $Y$ (respectively, of a neighborhood $N$ of $A$ into $Y$ ) such that $\left.\tilde{f}\right|_{A}=f$. A map is a continuous function.

First we state a lemma which demonstrates some difference between $\mathcal{H}_{c-o}(M)$ and $\mathcal{H}_{m}(M)$. The proof is rather straightforward and will be omitted. Let $\sigma=n$-simplex and $K_{0}=0$-simplices of $\sigma$.

Lemma 1. Given any map $f: K_{0} \rightarrow \mathcal{H}(M)$, where $\mathcal{H}(M)$ is given either the c-o or the majorant topology, such that for any $m \in M$, the convex ball $\left\langle f\left(K_{0}\right)(m)\right\rangle \subset M$. Then the convex extension $\tilde{f}: \sigma \rightarrow \bigodot_{\mathrm{c}-\mathrm{o}}(M)$ is continuous, where $\tilde{f}$ is defined as follows: For any $x=\sum_{i} t_{i} v_{i} \in \sigma$ and for any $m \in M$,

$$
\tilde{f}(x)(m)=\sum_{i} t_{i} f\left(v_{i}\right)(m) .
$$

REMARK. In general, $\tilde{f}: \sigma \rightarrow \bigodot_{m}(M)$ is not continuous.

THEOREM 2. $\mathcal{H}_{\mathrm{c}-\mathrm{o}}\left(l_{2}\right) \in \operatorname{AE}(\mathscr{N})$.

Remark. This cannot in general be extended to Hilbert manifolds. A counterexample is given in Example 2 that follows.

Proof. To simplify notation, we denote $\mathcal{C}_{\mathrm{c}-\mathrm{o}}\left(l_{2}\right)=\mathcal{C}(\boldsymbol{M})$ and $\mathcal{H}_{\mathrm{c}-\mathrm{o}}\left(l_{2}\right)=\mathcal{H}(\boldsymbol{M})$. Let $A$ be a closed subset of a metric space $X$ and let $h: A \rightarrow \mathcal{H}(M)$ be a given map. Using convex extension, the procedure of Dugundji [Du, p. 188] easily establishes that $\mathcal{C}(M) \in \mathrm{AE}(\mathfrak{N})$. Here some explanation is needed. First, convex ball of any $n$-points set is always well defined in $l_{2}$. Secondly, by Lemma 1 the convex extension yields a continuous function under (coarse) c-o topology.

Hence there is a map $\tilde{h}: X \rightarrow C(M)$ extending $h$. Our goal is to replace $\tilde{h}$ by another extension $H: X \rightarrow \mathcal{H}(M)$.

For each $x \in X-A$, let $B(x)$ be an open (metric) ball in $X-A$ with radius $<\frac{1}{2} d(x, A)$, where $d$ is a metric on $X$. Let $\mathcal{Q}$ be a nbd-finite open covering of $X-A$ such that the star of $\mathcal{U}$ refines the collection $\{B(x): x \in X-A\}$. For each $u \in \mathcal{U}$, choose a point $x_{u} \in \mathcal{U}$ and associate a point $a_{u} \in A$ such that $d\left(x_{u}, a_{u}\right)<2 d\left(x_{u}, A\right)$. Let $\left\{\lambda_{u}\right\}$ be a partition of unity subordinated on $\mathcal{Q}$.

We first replace $\tilde{h}: X \rightarrow \mathcal{C}(M)$ by the map $h^{\prime}: X \rightarrow \mathcal{C}(M)$ as follows:

$$
h^{\prime}(x)(m)= \begin{cases}\Sigma_{u \in \mathcal{Q}} \lambda_{u}(x) \tilde{h}\left(a_{u}\right)(m), & \text { if } x \in X-A, \\ h(x)(m), & \text { otherwise. }\end{cases}
$$

It is clear that $h^{\prime}$ is well defined and under the c-o topology, is a continuous extension over $A$. Let $K$ denote the nerve of $\mathscr{U}$ and let $p: X-A \rightarrow K$ be the usual map into its nerves; that is, $p(x)=\Sigma_{u \in \mathcal{Q}} \lambda_{u}(x) u$, where $\{u\}_{u \in \mathcal{U}}$ represents the vertices in $K$. Define $q: K \rightarrow \mathcal{C}(M)$ by the formula that, for any $y=\sum t_{u} u \in K$, $\sum t_{u}=1, q(y)(m)=\sum t_{u} h\left(a_{u}\right)(m)$. Clearly we have $q p=h^{\prime}$. Our strategy is to 
replace $q$ by another map $q^{\prime}: K \rightarrow \mathcal{H}(M)$ in such a way that $q^{\prime} p: X-A \rightarrow \mathcal{H}(M)$ can be extended to all $X$ agreeing with $h$ on $A$.

Let $K_{i}$ denote the $i$ th-skeleton of $K$. In the following we will define a sequence of maps $r_{0}, r_{1}, r_{2}, \ldots$ such that $r_{i}: K_{i} \rightarrow \mathcal{H}(M)$ and $r_{i}$ extends $r_{i-1}$ over $K_{i-1}$. The map we wanted will be the limiting map on $K=\cup K_{i}$.

By hypothesis $q\left(K_{0}\right) \subset \mathcal{H}(M)$. Define $r_{0}$ by $r_{0}=q \mid K_{0}$. For any 1 -simplex $\sigma \in K_{1}$ and any $m \in M, q(\sigma)(m)$ is a convex segment in $M\left(=l_{2}\right)$. Let $N(\sigma, m)$ be an $\varepsilon$-neighborhood about $q(\sigma)(m)$, where $\varepsilon=\frac{1}{2} \operatorname{Mesh}(q(\sigma)(m))$. Now fix any $\sigma \in K_{1}$, by Theorem 1 and by the fact that $N(\sigma, m)$ is a convex body, $\left.q\right|_{\partial \sigma}$ is homotopic to a map $r_{1}: \sigma \rightarrow \mathcal{H}(M)$ by a homotopy $\tilde{q}: \sigma \times I \rightarrow \mathcal{C}(M) \operatorname{rel}(\partial \sigma)$ such that $\tilde{q}(\sigma \times I)(m)$ $\subset N(\sigma, m)$ for any $m \in M$. Thus $r_{1}\left|\partial \sigma=r_{0}\right| \partial \sigma$. In the c-o topology, the extension $r_{1}$ and the subsequent homotopy are continuous. Repeating this to every $\sigma \in K_{1}$ we obtain a map $r_{1}: K_{1} \rightarrow \mathcal{H}(M)$ which is homotopic to $q \mid K_{1}$ by a homotopy $\tilde{q}: K_{1} \times$ $I \rightarrow \mathcal{C}(M) \operatorname{rel}\left(K_{0}\right)$ such that for any l-simplex $\sigma$, and for any $m \in M, \tilde{q}(\sigma \times I)(m)$ $\subset N(\sigma, m)$.

By reparametrizing $K$ we can use the homotopy $\tilde{q}$ to obtain a new map $q_{1}: K \rightarrow$ $\varrho(M)$ with the property that for any $\sigma \in K$ and for any $m \in M, q_{1}(\sigma)(m)$ is contained in the $\varepsilon$-neighborhood of $q(\sigma)(m)$ with $\varepsilon=\frac{1}{2} \operatorname{Mesh}(q(\sigma)(m))$.

Replacing $q$ by $q_{1}$ we can duplicate the above procedure to obtain a map $r_{2}: K_{2} \rightarrow \mathcal{H}(M)$ which extends $r_{1}$ over $K_{1}$ and a map $q_{2}: K \rightarrow \mathcal{C}(M)$ such that $q_{2} \mid K_{2}=r_{2}$ and that for any $\sigma \in K, m \in M, q_{2}(\sigma)(m)$ is contained in the $\varepsilon$-neighborhood of $q(\sigma)(m)$, where $\varepsilon=\left(1 / 2+1 / 2^{2}\right) \operatorname{Mesh}(q(\sigma)(m))$.

Inductively we obtain a sequence of maps $\left\{r_{i}\right\}$ and $\left\{q_{i}\right\}$ with $r_{i}: K_{i} \rightarrow \mathcal{H}(M)$ and $q_{i}: K \rightarrow \mathcal{C}(M)$ such that $r_{i}\left|K_{i-1}=r_{i-1}, q_{i}\right| K_{i}=r_{i}$ and for any $\sigma \in K, m \in M$, $q_{n}(\sigma)(m)$ is contained in the $\varepsilon$-neighborhood of $q(\sigma)(m)$ with

$$
\varepsilon=\left(1 / 2+1 / 2^{2}+\cdots+1 / 2^{n}\right) \operatorname{Mesh}(q(\sigma)(m)) .
$$

Define $q^{\prime}: K \rightarrow \mathcal{H}(M)$ by $q^{\prime} \mid K_{i}=r_{i}$. Then we have, for $\sigma \in K, m \in M, q^{\prime}(\sigma)(m)$ is contained in the $\varepsilon$-neighborhood of $q(\sigma)(m)$ with

$$
\varepsilon=\operatorname{Mesh}\left(q^{\prime}(\sigma)(m)\right) \leqslant 3 \operatorname{Mesh}(q(\sigma)(m)) .
$$

Define $H: N \rightarrow \mathcal{H}(M)$ by

$$
H(x)= \begin{cases}q^{\prime} p(x), & \text { if } x \in N-A, \\ h(x), & \text { otherwise. }\end{cases}
$$

It is straightforward to verify that $H$ is a desired extension of $h$.

EXAMPLE 2. $\mathscr{H}_{\mathrm{c}-\mathrm{o}}(M) \notin \mathrm{ANE}(\mathfrak{N})$.

Let $M=Y \times l_{2}$ be given as in Example 1 and consider $X=[0,1]$ and $A=\{0\} \cup$ $\{1 / n\} \subset X$. Let $\mathcal{C}_{i}$ denote the $i$ th circle on $Y$. Define a map $h: A \rightarrow \mathcal{H}_{\mathrm{c}-\mathrm{o}}(M)$ by $h(1 / i)=f_{i} \times \mathrm{id}$, where $f_{i}$ is the homeomorphism on $Y$ by interchanging the upper and lower circles of all $c_{j}, j \geqslant i$, and leaves the remaining space unchanged. Moreover, define $h(0)=$ identity on $M$. Since any neighborhood of the identity in $\mathcal{H}_{\mathrm{c}-\mathrm{o}}(M)$ contains all but finitely many $f_{i} \times \mathrm{id}, h$ is continuous (caution, $h$ is not continuous when considering it from $A$ into $\mathcal{K}_{m}(M)$ ). On the other hand, since any neighborhood $N$ of 0 in $X$ contains all but finitely many members of $A$ (and the intervals between them), it is clear that $h$ has no continuous extension to $N$. 
The fact that $h$ is not continuous when $\mathcal{H}(M)$ is given the majorant topology is important since the following theorem shows that if a map $h: A \rightarrow \mathcal{H}_{m}(M)$ is continuous, then $h$ has a continuous extension to a neighborhood of $A$ into $\mathcal{H}_{\mathrm{c}-\mathrm{o}}(M)$.

TheOREM 3. Given a closed set $A$ in a metric space $X$ and given a map $h: A \rightarrow$ $\mathcal{H}_{m}(M)$, there is a neighborhood $N$ of $A$ and a continuous extension $\tilde{h}: N \rightarrow \mathcal{H}_{\mathrm{c}-\mathrm{o}}(M)$.

REMARK. The extension is not valid in the space $\mathcal{H}_{m}(M)$ (see Example 3 below).

Proof. By Lemma 1 and the convex extension defined there, the usual procedure of Dugundji [Du, p. 188] easily implies that $\bigodot_{\mathrm{coo}}(M) \in \operatorname{ANE}(\mathfrak{N})$. Hence there is a neighborhood $\tilde{N} \supset A$ and a map $h^{\prime}: \tilde{N} \rightarrow \bigodot_{\text {c-o }}(M)$ extending $h$. Recall that by [He] we may assume $M$ is an open set in $l_{2}$. Let $\mathcal{O}, \mathcal{V}$ be open coverings of $M$ consisting of convex sets such that each $v \in \mathcal{V}$ is some $\varepsilon$-neighborhood $N(x, \varepsilon)$ and that the collection $\{N(x, 3 \varepsilon)\}$ is a star refinement of $\theta$.

Since $h: A \rightarrow \mathcal{H}(M)$ is continuous in the majorant topology (this is where the majorant topology comes into play), we have, for each $a \in A$, a neighborhood $N(a)$ of $a$ in $\tilde{N}$ such that for any $m \in M, h^{\prime}(N(a)(m)) \subset v$ for some $v \in \mathcal{V}$. Denote $N=\cup_{a \in A} N(a) \subset \tilde{N}$. We wish to replace $\left.h^{\prime}\right|_{N}$ by another extension $\tilde{h}: N \rightarrow$ $\mathcal{H}_{m}(M)$. From here on the remaining argument mimics the proof of Theorem 2 word for word and therefore will be omitted.

EXAMPlE 3. $\mathcal{H}_{m}\left(l_{2}\right) \notin \operatorname{ANE}(\mathfrak{N})$.

This is a consequence of the following facts:

(a) $\mathcal{H}_{m}\left(l_{2}\right)$ is totally disconnected;

(b) there is an $h_{n} \in \mathcal{H}_{m}\left(l_{2}\right) \backslash\{e\}$ such that $h_{n} \rightarrow e$, where $e=$ identity.

Proof. (a) For $f, g \in \mathcal{H}_{m}\left(l_{2}\right), f \neq g$, take $x_{n} \in l_{2}$ with $\left\|f\left(x_{n}\right)-g\left(x_{n}\right)\right\|>0$ and $\left\{x_{n}\right\}$ is closed. Let $\varepsilon: l_{2} \rightarrow(0, \infty)$ be such that $\varepsilon\left(x_{n}\right)=\left\|f\left(x_{n}\right)-g\left(x_{n}\right)\right\| / n$. Consider $V=\cup_{c>0}\left\{h \in \mathcal{H}_{m}\left(l_{2}\right):\|h(x)-f(x)\|<c \cdot \varepsilon(x)\right\}$. We have $f \in V$ and $g \notin V$, but $V$ is both open and closed.

(b) Take $x_{n} \rightarrow 0, x_{n} \neq 0$ and $h_{n} \in \mathcal{H}_{m}\left(l_{2}\right)$ such that $h_{m}\left(x_{n}\right)=0$ and $h_{n}=$ identity outside of $N_{n}=\left\{x:\|x\|<2\left\|x_{n}\right\|\right\}$. Now, let $x=[0,1]$ and $A=\{0\} \cup\{1 / n\}$. The map $h: A \rightarrow \mathcal{H}_{m}\left(l_{2}\right)$ defined by $h(0)=$ identity and $h(1 / n)=h_{n}$ clearly has no continuous extension to any neighborhood of $A$ into $\mathcal{H}_{m}\left(l_{2}\right)$ (see also Example 2).

REMARK. The above example and its proof was communicated to me by $\mathrm{T}$. Dobrowolski.

4. Lemmas. In this section we prove two lemmas which are needed in $§ 5$. In what follows, all spaces considered will be metric spaces. Given a homotopy $f=\left\{f_{t}\right\}: A$ $\rightarrow Y$, let $I_{a}$ denote the orbit $\left\{f_{t}(a)\right\}_{t}$ over $a$. We say a collection of open sets $\{N(a)\}_{a \in A}$ in $Y$ is an $f$-assignment (for the images of $f$ ) provided the following are true. (1) For each $a \in A, I_{a} \in N(a)$, (2) there are neighborhoods $U(a) \ni a, V(a) \supset$ $I_{a}$ such that $V(a) \subset N(a)$ and for any $b \in U(a), I_{b} \subset V(a) \subset N(b)$.

The concept of $f$-assignment is quite essential in the proof of Theorem 1. It briefly says that each $f_{t}$-orbit is assigned an open neighborhood $N(a)$ and that the size of $N(a)$ varies continuously with $a$. It turns out that the collection $\{N(a)\}$ can be assigned quite canonically if we consider, for instance, $\varepsilon$-neighborhoods of $I_{a}$ 's in $Y$ and where $\varepsilon$ depends continuously with the diameter of $I_{a}$. This turns out to be exactly the scheme we will employ later on. 
A closed set $A_{0} \subset A$ is a Z $\mathbf{Z}$-set in $A$ provided that for any open covering $\mathscr{Q}$ of $A$, there is a map $f: A \rightarrow A-A_{0}$ which is W-close to the identity. A map $f: X \times Y \rightarrow \mathbf{Z}$ $\times Y$ is $Y$-preserving provided $f$ preserves the $Y$-coordinate of each point. If $p_{i}: X_{i} \times$ $Y \rightarrow Y$ are product bundles, $i=1,2$, where $p_{i}$ 's are the projections, we also say that $f: X_{1} \times Y \rightarrow X_{2} \times Y$ is a fibre map provided $f$ is $Y$-preserving, that is, $p_{2} f(x, y)=$ $p_{1}(x, y)$ for any $(x, y)$. In the following, let $s$ denote $(-1,1)^{\infty}$ and let $p: M \times B^{n} \rightarrow$ $B^{n}$ be the projection.

LEMMA 2 (MAPPING REPLACEMENT). Let $A_{0}$ be a closed set in a complete separable metric space $A$. Suppose $f\left(=\left\{f_{t}\right\}\right): A \times I \rightarrow M \times B^{n}$ is a given homotopy such that $f_{0}, f_{1}$ are closed imbeddings, $I_{a}=\{$ point $\}$ for all $a \in A_{0}, f_{0}(A) \cap f_{1}(A)=f\left(A_{0} \times I\right)$ and $f(A \times I) \cap(M \times\{b\})$ is a closed Z-set in each $b \in B^{n}$. Furthermore, suppose that $\{N(a)\}_{a \in A-A_{0}}$ is an f-assignment for $f\left(\left(A-A_{0}\right) \times I\right)$. Then there is a homotopy $g\left(=\left\{g_{t}\right\}\right): A \times I \rightarrow M \times B^{n}$ such that

(1) $g \mid(A \times\{0,1\}) \cup\left(A_{0} \times I\right)=f$,

(2) $g(A \times I)$ is closed and $g(A \times I) \cap(M \times\{b\})$ is a Z-set in each $M \times\{b\}$,

(3) $p g(a, t)=p f(a, t)$ for all $(a, t)$,

(4) each $g_{t}$ is an imbedding and $g_{t}(A) \cap g_{r}(A)=g_{0}\left(A_{0}\right)$ whenever $t \neq r$ and

(5) $g(\{a\} \times I) \subset N(a)$ for all $a \in A-A_{0}$.

Proof. Lemma 2 is similar to the mapping replacement lemma in [Ch-Wo II], with the exception of (5). To establish condition (5) of the lemma, we repeat the basic idea behind the original argument, which goes as follow.

By Theorem 2 of [Ch-Wo II, p. 263] there is a $B^{n}$-preserving homeomorphism of $M \times B^{n}$ onto $(s \times M) \times B^{n}$ sending $f(A \times I)$ into $\{0\} \times M \times B^{n}$ where $0=$ $(0,0, \ldots) \in s$. So, to simplify notation, we will replace $M \times B^{n}$ by $(s \times M) \times B^{n}$ and assume $f(A \times I) \subset\{0\} \times M \times B^{n}$. For each $a \in A-A_{0}$, let $U(a) \ni a$ and $V(a) \subset N(a)$ be associated coverings given by the definition of $f$-assignment.

For each $a \in A-A_{0}, I_{a}$ is contained in a box-like neighborhood $R(a) \times S(a) \subset$ $V(a)$, where $S(a)$ is an open set in $M \times B^{n}$ and $R(a)$ is a basic neighborhood in $s$ of the form $\Pi_{i \geqslant 1}\left(-t_{i}(a), t_{i}(a)\right)$. Since $\{a\} \times I$ is compact, we may assume $f(U(a) \times I)$ $\subset\{0\} \times S(a)$. Let $\{W(a)\}$ be a locally finite refinement of $\{U(a)\}$ such that $W(a) \subset U(a)$ for all $a \in A-A_{0}$ (we caution that $W(a)$ may no longer contain $a$ ). For each fix $i \geqslant 1$, define $\lambda_{i}: A-A_{0} \rightarrow(0,1)$ by $\lambda_{i}(x)=\operatorname{Min}\left\{t_{i}(a): x \in W(a)\right\}$. Clearly $\lambda_{i}$ is locally bounded above zero. It is well known that there is a continuous real-valued map $\mu_{i}: A-A_{0} \rightarrow(0,1)$ such that $0<\mu_{i}(x)<\lambda_{i}(x)$.

Now for any $a \in A-A_{0}, a \in W(b) \subset U(b)$ for some $b$. Hence $I_{a} \subset\{0\} \times S(b)$. Furthermore, if for all $i \geqslant 1,0 \leqslant t_{i} \leqslant \mu_{i}(a)$, then $t_{i}<\lambda_{i}(a) \leqslant t_{i}(b)$. Hence the set $K(a) \subset R(b) \times S(b)$, where

$$
K(a)=\left\{\left(\left(t_{i}\right), m^{\prime}, b^{\prime}\right):\left(0, m^{\prime}, b^{\prime}\right) \in I_{a}, 0 \leqslant t_{i} \leqslant \mu_{i}(a)\right\} .
$$

Let $h: A \times I \rightarrow s$ be an imbedding onto a closed Z-set such that all $\pi_{i} h(a, t)>0$, where $\pi_{i} h(a, t)$ is the $i$ th-coordinate of $h(a, t)$. Let $\lambda: A \times I \rightarrow I$ be a map such that $\lambda^{-1}(0)=(A \times\{0,1\}) \cup\left(A_{0} \times I\right)$. Define $g: A \times I \rightarrow s \times M \times B^{n}$ by $g((a, t))=$ $\left(\left(t_{i}\right), m, b\right)$, where

$$
(0, m, b)=f((a, t)) \quad \text { and } \quad t_{i}=\lambda(a, t) \cdot \mu_{i}(a) \cdot \pi_{i} h(a, t) .
$$


It is routine to check that $g$ is a desired mapping for the lemma. In particular, to verify condition (5), we have $g((a, t))=\left(\left(t_{i}\right)_{i}, m, b\right)$, where $(a, t) \in\{a\} \times I$. Each $t_{i} \leqslant \mu_{i}(a)$. Since $a \in W(b) \subset U(b)$ for some $W(b), g(\{a\} \times I) \subset K(a) \subset R(b) \times$ $S(b) \subset V(b)$. By condition (2) of the hypothesis of $f$-assignment, $V(b) \subset N(a)$. Hence $g(\{a\} \times I) \subset N(a)$.

LEMMA 3. There is a level-preserving imbedding $f: M \times I \rightarrow M \times I$ such that for any $t<1, f \mid M \times\{t\} \in \mathcal{H}(M)$ and $f(M \times\{1\})$ is a closed Z-set in $M \times\{1\}$.

Proof. By [An-Sc], $M$ is homeomorphic to $M \times s$. So we may replace $M$ by $M \times s$. Fix an $a \in(0,1)$. Let $\mu=\left\{\mu_{t}\right\}$ be a homotopy on $[-1,1] \operatorname{rel}(\{-1,1\})$ such that $\mu_{0}=$ identity, for any $t<1, \mu_{t}$ is a homeomorphism on $[-1,1]$ sending $[-a, a]$ onto $\left[-a_{t}, a_{t}\right]$, where $a_{t}=(1-t) a+t$ and $\mu_{1}$ maps $(-a, a)$ homeomorphically onto $(-1,1)$ while collapsing $[-1,-a]$ onto $-1,[a, 1]$ onto 1 . Define $g:(M \times s) \times$ $I \rightarrow(M \times s) \times I$ by

$$
g\left(m,\left(t_{i}\right), t\right)=\left(m,\left(\mu_{t}^{-1}\left(t_{i}\right)\right), t\right) .
$$

Note that $\mu_{t}^{-1}(-1,1)=(-1,1)$ for $t<1$ and $\mu_{1}^{-1}(-1,1)=(-a, a)$. Hence

$$
g(M \times s \times\{1\})=M \times(-a, a)^{\infty} \times\{1\}
$$

and for $t<1, g \mid M \times s \times\{t\}$ is a homeomorphism. The closure of $g(M \times s \times\{1\})$ in $M \times s \times\{1\}$ is $K=M \times[-a, a]^{\infty} \times\{1\}$. It is well known that

$$
K-g(M \times s \times\{1\}) \quad\left(=M \times\left([-a, a]^{\infty}-(-a, a)^{\infty}\right) \times\{1\}\right)
$$

is a countable union of compact Z-sets in $M \times s \times\{1\}$. So by [Ch-Wo II] there is a level-preserving homeomorphism $h$ of $(M \times s) \times I$ onto $(M \times s-K) \times I$. Let $f=h g$. Then $f(M \times s \times\{1\})$ is a closed Z-set in $M \times s \times\{1\} . f$ is what we wanted.

Lemma 3 can be easily generalized to

LEMMA 4. There is a $B^{n}$-preserving imbedding $f: M \times B^{n} \rightarrow M \times B^{n}$ such that for any $b \in \operatorname{Int}\left(B^{n}\right), f \mid M \times\{b\}$ is a homeomorphism and $f(M \times\{b\})$ is a closed Z-set in $M \times\{b\}$ for each $b \in S^{n-1}$.

5. Proof of Theorem 1. First we assume that for some $b_{0} \in S^{n-1}, h \mid M \times\left\{b_{0}\right\}=$ identity on $M . h$ is fibred homotopic to the identity by $\phi=\left\{h_{t}\right\}$ defined by

$$
h_{t}(m, b)=\left(q h\left(m,(1-t) b_{0}+t b\right), b\right) .
$$

If $q h\left(\{m\} \times B^{n}\right)=$ point $\}$ for some $m$, then $q h\left(\{m\} \times B^{n}\right)=\{m\}$ since $q h\left(m, b_{0}\right)=m$. Thus $h(m, b)=(m, b)$ for all $b \in B^{n}$. Let

$$
M_{0}=\left\{m \in M: q h\left(\{m\} \times B^{n}\right)=\{m\}\right\} .
$$

$M_{0}$ is closed. It is clear that $\left\{h_{t}\right\}$ is a homotopy relative $M_{0} \times B^{n}$. Furthermore, if $m \notin M_{0}$, then $\operatorname{Mesh}\left(q h\left(\{m\} \times B^{n}\right)\right)>0$ and $N(m)$ (as defined in the theorem) is an open neighborhood of $q h\left(\{m\} \times B^{n}\right)$. Let $M_{1}=M-M_{0}$. We now divide the remaining arguments into four steps.

Step 1. The purpose is to establish the homotopy $\mu=\left\{\mu_{t}\right\}$.

By Lemma 4 there is a closed fibred imbedding $g: M \times B^{n} \rightarrow M \times B^{n}$ such that $q \mid M \times \operatorname{Int}\left(B^{n}\right)$ is a homeomorphism onto $M \times \operatorname{Int}\left(B^{n}\right)$ and $g(M \times\{b\})$ is a 
closed Z-set in $M \times\{b\}$ for $b \in S^{n-1}$. Denote

$$
B=g\left(M \times S^{n-1}\right), \quad B_{0}=g\left(M_{0} \times S^{n-1}\right), \quad B_{1}=B-B_{0} .
$$

For $m \in M_{1}$, let $O(m)$ denote an open set in $M \times B^{n}$ such that $g^{-1}(O(m))=N(m)$ $\times B^{n}$. Let $\phi^{\prime}=\left\{h_{t}^{\prime}\right\}: g\left(M \times B^{n}\right) \rightarrow g\left(M \times B^{n}\right)$ denote the induced homotopy $\left(\right.$ rel $\left.g\left(M_{0} \times B^{n}\right)\right)$ defined by $h_{t}^{\prime}=g h_{t} g^{-1}$.

For each $a^{\prime} \in g\left(M_{1} \times B^{n}\right)$, we assign an open neighborhood $N_{1}\left(a^{\prime}\right)$ as follows: For some $m \in M_{1}, g^{-1}\left(a^{\prime}\right) \in\{m\} \times B^{n} \subset N(m) \times B^{n}$. So

$$
\left\{h_{t}^{\prime}\left(a^{\prime}\right)\right\}_{t} \subset g\left(N(m) \times B^{n}\right) \subset O(m) .
$$

Let $N_{1}\left(a^{\prime}\right)=O(m)$. We check readily that $\left\{N_{1}\left(a^{\prime}\right)\right\}_{a^{\prime} \in g\left(M_{1} \times B^{n}\right)}$ is a $\phi^{\prime}$-assignment. It follows that there are neighborhoods $U\left(a^{\prime}\right) \ni a^{\prime}, V\left(a^{\prime}\right) \supset\left\{h_{t}^{\prime}\left(a^{\prime}\right)\right\}_{t}$ such that $U\left(a^{\prime}\right)$ $\subset V\left(a^{\prime}\right) \subset N_{1}\left(a^{\prime}\right)$ and for any $b \in U\left(a^{\prime}\right),\left\{h_{t}^{\prime}\left(a^{\prime}\right)\right\}_{t} \subset V\left(a^{\prime}\right) \subset N_{1}(b)$.

By [Ch-Wo II, Theorem 1] there is a fibred isotopy $\lambda=\left\{\lambda_{t}\right\}: M \times B^{n} \rightarrow M \times B^{n}$ (rel $g\left(M_{0} \times B^{n}\right)$ ) such that $\lambda_{0}=$ identity, $\lambda_{1}\left(B_{1}\right) \cap h_{1}^{\prime}\left(B_{1}\right)=\phi$ and $\left\{\lambda_{t}\right\}$ is limited by $\left\{U\left(a^{\prime}\right)\right\}$. Composing $\left\{\lambda_{t}^{-1}\right\}$ with $\left\{h_{t}^{\prime}\right\}$ (one after the other) we obtain a homotopy

$$
\mu=\left\{\mu_{t}\right\}: \lambda_{1} g\left(M \times B^{n}\right) \rightarrow h_{1}^{\prime} g\left(M \times B^{n}\right) \quad\left(\operatorname{rel} g\left(M_{0} \times B^{n}\right)\right)
$$

such that $\mu_{0}=\lambda_{0}^{-1}=$ identity,

$$
\mu_{1 / 2}=\lambda_{1}^{-1}: \lambda_{1} g\left(M \times B^{n}\right) \rightarrow g\left(M \times B^{n}\right)
$$

and

$$
\mu_{1}=h_{1}^{\prime} \cdot \lambda_{1}^{-1}: \lambda_{1} g\left(M \times B^{n}\right) \rightarrow h_{1}^{\prime} g\left(M \times B^{n}\right) .
$$

Denote

$$
A=\lambda_{1}(B), \quad A_{0}=\lambda_{1}\left(B_{0}\right), \quad A_{1}=A-A_{0} .
$$

Restricting $\left\{\mu_{t}\right\}$ on $A$, we observe that (1) $\mu_{0}$ and $\mu_{1}$ are closed imbeddings of $A$ into $M \times B^{n}$, (2) $\mu_{0}\left(A_{1}\right) \cap \mu_{1}\left(A_{1}\right)=\lambda_{1}\left(B_{1}\right) \cap h_{1}^{\prime}\left(B_{1}\right)=\phi,(3) \mu_{0}(A) \cap \mu_{1}(A)=A_{0}$ since $\mu_{t} \mid A_{0}=$ identity and (4) each $\mu(A \times I) \cap(M \times\{b\})$ is a closed Z-set in $M \times\{b\}$. Furthermore, for any $a^{\prime} \in g\left(M \times B^{n}\right),\left\{\lambda_{t}\left(a^{\prime}\right)\right\}_{t} \subset U(b)$ for some $b$. Hence $\left\{\lambda_{t}\left(a^{\prime}\right)\right\}_{t} \subset U(b) \subset V(b) \subset N_{1}\left(a^{\prime}\right)$. It follows that $\left\{\mu_{t}\left(\lambda_{1}\left(a^{\prime}\right)\right)\right\}_{t} \subset N_{1}\left(a^{\prime}\right)$. For $a \in A_{1}$, let $a^{\prime}=\lambda_{1}^{-1}(a)$ and $N^{\prime}(a)=N_{1}\left(a^{\prime}\right)$. Then $\left\{\mu_{t}(a)\right\}_{t} \subset N^{\prime}(a)$. We easily verify that $\left\{N^{\prime}(a)\right\}$ is a $\mu$-assignment for $\mu\left(A_{1} \times I\right)$. By the Mapping Replacement Lemma (Lemma 2) we may further assume that $\mu(A \times I)$ is closed, each $\mu_{t}$ is an imbedding such that $\mu_{t}(A) \cap \mu_{r}(A)=A_{0}$ whenever $t \neq r$.

Step 2. The purpose is to straighten out $\left\{\mu_{t}(A)\right\}$ so that we can move $\mu_{0}\left(A_{1}\right)$ onto $\mu_{1}\left(A_{1}\right)$ (rel $A_{0}$ ) by an ambient fibre isotopy. The delicate details are for the purpose of keeping track of the size of the isotopy.

By virtue of [Ch-Wo II, Corollary 2] there is a $B^{n}$-preserving open imbedding $\sigma: M \times B^{n} \rightarrow(0,1) \times s \times B^{n}$ such that $\sigma(\mu(A \times I))$ is closed in $(0,1) \times s \times B^{n}$ and that $\sigma(\mu(A \times I)) \cap(0,1) \times s \times\{b\}$ is a Z -set in each $(0,1) \times s \times\{b\}$. Let $\nu_{0}$ be an imbedding of $A$ into $s$ such that $\nu_{0}(A)$ is a closed Z-set in $s$. Let $r: A \rightarrow\left[0, \frac{1}{3}\right]$ be a map such that $r^{-1}(0)=A_{0}$. Define $v: A \times I \rightarrow(0,1) \times s \times B^{n}$ by

$$
v(a, t)=\left(\frac{1}{3}+r(a) t, \nu_{0}(a), p(\mu(a, t))\right) \text {. }
$$


(Recall that $p: M \times B^{n} \rightarrow B^{n}$ is the projection.) Next we map $\sigma \mu(A \times I)$ onto $\nu(A \times I)$ by the $B^{n}$-preserving homeomorphism $\rho$ defined by $\rho(\sigma \mu(a, t))=\nu(a, t)$

$$
\begin{array}{ccc}
A \times I & \stackrel{\mu}{\rightarrow} & \mu(A \times I) \\
\downarrow v & & \downarrow \sigma \\
v(A \times I) & \stackrel{\rho}{\leftarrow} & \sigma \mu(A \times I) \\
\cap & & \cap \\
(0,1) \times s \times B^{n} & \stackrel{\tilde{\rho}}{\leftarrow} & (0,1) \times s \times B^{n} .
\end{array}
$$

Since any two maps in $s$ are homotopic, by [Ch-Wo II, Theorem 5] $\rho$ extends to a $B^{n}$-preserving homeomorphism $\tilde{\rho}$ of $(0,1) \times s \times B^{n}$ onto itself.

Step 3. The main idea now is to map $\nu(A \times\{0\})$ onto $\nu(A \times\{1\})\left(\operatorname{rel} \nu\left(A_{0} \times I\right)\right)$ via a $B^{n}$-preserving ambient isotopy on $(0,1) \times s \times B^{n}$. The delicate detail is to assure each fibre of the isotopy lives within its required neighborhood.

Let $k_{1}=\tilde{\rho} \sigma$ and $k=k_{1} \lambda_{1} g$.

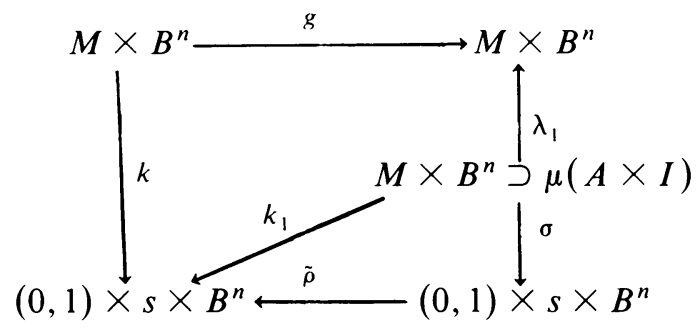

Thus $k_{1}(\mu(A \times I))=\nu(A \times I)$. Denote $\tilde{N}(a)=k_{1}\left(N^{\prime}(a)\right)$, where $a \in A_{1}$ (see Step 1). $\tilde{N}(a)$ is a $\nu$-assignment for $\nu\left(A_{1} \times I\right)$. Since each fibre $\nu(\{a\} \times I)$ is essentially a line segment in $(0,1) \times s \times B^{n}$, by virtue of $\nu$-assignment, there are box-like neighborhoods $\{B(a)\}_{a \in A_{1}}$.

$$
B(a)=I(a) \times S(a) \supset \nu(\{a\} \times I),
$$

where $I(a)=\left(t_{1}(a), t_{2}(a)\right)$ is an open interval containing $\left[\frac{1}{3}, \frac{1}{3}+r(a)\right]$ and $S(a)$ is an open neighborhood in $s \times B^{n}$ such that $B(a) \subset \tilde{N}(a)$, and more importantly,

$$
B\left(a^{\prime}\right) \subset \tilde{N}(a) \text { whenever } \nu(a, 0) \in B\left(a^{\prime}\right) .
$$

Since $k\left(M_{0} \times B^{n}\right)$ is closed and disjoint from $\nu\left(A_{1} \times I\right)$, we may require that $B(a) \cap k\left(M_{0} \times B^{n}\right)=\varnothing$.

Let $\left\{S^{\prime}(a)\right\}$ be a locally finite refinement of $\{S(a)\}$ such that $S^{\prime}(a) \subset S(a)$ for all $a$. Denote $B^{\prime}(a)=I(a) \times S^{\prime}(a)$ (we caution that $B^{\prime}(a)$ may no longer contain $\nu(a, 0)) .\left\{k^{-1}\left(B^{\prime}(a)\right)\right\}_{a \in A_{1}}$ is a locally finite covering of $M_{1} \times S^{n-1}$ in $M_{1} \times B^{n}$. Let $c=$ center of $B^{n}$. Treating $M_{1} \times\left(B^{n}-c\right)$ as $M_{1} \times S^{n-1} \times[0,1)$, it is well known that there is a map $r_{1}: M \times S^{n-1} \rightarrow[0,1)$ such that $r_{1}^{-1}(0)=M_{0} \times S^{n-1}$ and for $x=(m, b)=k^{-1}(\nu(a, 0)) \in M_{1} \times S^{n-1}$, the set

$$
l(a)=\left\{(m,(1-t) b+t c): t \leqslant r_{1}(x)\right\} \subset k^{-1}\left(B^{\prime}(\tilde{a})\right)
$$


for some $k^{-1}\left(B^{\prime}(\tilde{a})\right)$, where $(1-t) b+t c$ is a point on the line segment between $b \in S^{n-1}$ and the center $c$ of $B^{n}$. Thus $k(l(a)) \subset B^{\prime}(\tilde{a})$. Let

$$
L=U\left\{l(a): a \in A, k^{-1} \nu(a, 0) \in M_{1} \times S^{n-1}\right\} .
$$

Then $L$ is a neighborhood of $M_{1} \times S^{n-1}$ in $M_{1} \times B^{n}$. Since we are dealing with $B^{n}$-preserving mappings, $\lambda_{1} g(L)$ is a neighborhood of $M \times S^{n-1}$ in $M \times B^{n}$. But $\mu(A \times I) \subset M \times S^{n-1}$, hence $k(L)=k_{1}\left(\lambda_{1} g(L)\right)$ is a neighborhood of

$$
k_{1}(\mu(A \times I))=\nu(A \times I) .
$$

Using the local finiteness of $\left\{S^{\prime}(a)\right\}$ and the box-like neighborhoods $\left\{B^{\prime}(a)\right\}$, it is straightforward to show that there are mappings $q_{1}, q_{2}: s \times B^{n} \rightarrow(0,1)$ such that, for $i=1$ and 2 ,

(0) $q_{1}(x) \leqslant q_{2}(x)$ for all $x$,

(1) $q_{i}(x)=\frac{1}{3}$ whenever $x \in \cup_{a \in A_{1}} S^{\prime}(a)$, and

(2) $t_{1}(a)<q_{1}(x)<\frac{1}{3} \leqslant \frac{1}{3}+r(a)<q_{2}(x)<t_{2}(a)$,

whenever

$$
x \in S^{\prime}(a) \text { for some } a \in A_{1} .
$$

Moreover, we require that

(3) $\left[q_{1}(x), q_{2}(x)\right] \times\{x\} \subset k(L)$ whenever $q_{1}(x)<q_{2}(x)$.

(Condition (3) is to assure that the action takes place only in $k(L)$.)

By well-known technique there is a (linear) $s \times B^{n}$-preserving isotopy $\phi_{t}:(0,1) \times s$ $\times B^{n} \rightarrow(0,1) \times s \times B^{n}$ such that $\phi_{0}=$ identity, $\phi_{t}$ is the identity outside of $k(L)$, $\phi_{1}(\nu(a, 0))=\nu(a, 1)$ for all $a \in A$ and the motion of $\left\{\phi_{t}\right\}$ on each $(0,1) \times\{x\} \subset$ $(0,1) \times s \times B^{n}$ takes place in $\left[q_{1}(x), q_{2}(x)\right] \times\{x\}$.

Step 4 . The goal is to verify that $\phi_{1}$ induces the required homeomorphism $h^{\prime}$.

First we state three properties of $\phi_{1}$.

(i) $\phi_{1}\left(B^{\prime}(a)\right) \subset B^{\prime}(a)$ for all $a \in A_{1}$,

(ii) $\phi_{1}$ is the identity on $k\left(M_{0} \times B^{n}\right)$, and

(iii) $\phi_{1}$ is the identity outside of $k(L)$.

Recall that $k^{-1}\left(\nu\left(A_{i} \times\{0\}\right)\right)=M_{i} \times S^{n-1}$ for $i=0$ or 1 . For any $(m, b)=$ $k^{-1}(\nu(a, 0)), a \in A$, let

$$
R(a)=\left\{(m, t b+(1-t)) c: t \in[0,1], c=\operatorname{Center}\left(B^{n}\right)\right\}
$$

be the radius of $\{m\} \times B^{n}$ at $(m, b)$.

Now fix any $m \in M_{1}$ and consider some radius $R(a) \supset l(a)$, where $k^{-1}(\nu(a, 0))$ $=(m, b) \in\{m\} \times B^{n}$. By Step 3 above, $l(a) \subset k^{-1}\left(B^{\prime}\left(a^{\prime}\right)\right)$ for some $a^{\prime}$. So $k(l(a))$ $\subset B^{\prime}\left(a^{\prime}\right)$. But $(m, b) \in l(a)$, so $k(m, b)=\nu(a, 0) \in B^{\prime}\left(a^{\prime}\right) \subset B\left(a^{\prime}\right)$. By (*) of Step $3, B\left(a^{\prime}\right) \subset \tilde{N}(a)$. Hence $k(l(a)) \subset B\left(a^{\prime}\right) \subset \tilde{N}(a)$. By property (i) $\phi_{1}(k(l(a))) \subset \tilde{N}(a)$. Since $l(a)$ is any radius of $\{m\} \times B^{n}, \phi_{1}\left(k\left(\{m\} \times B^{n}\right)\right) \subset \tilde{N}(a)$.

Finally define $h^{\prime}: M \times B^{n} \rightarrow M \times B^{n}$ by $h^{\prime}=g^{-1} k_{1}^{-1} \phi_{1} k$. We claim that $h^{\prime}$ is what we wanted for the lemma. (We must first point out that, although it is natural to define $h^{\prime}=k^{-1} \phi_{1} k$, it is not a correct choice since $\phi_{1} k\left(M \times B^{n}\right)$ may not contain in $k\left(M \times B^{n}\right)$.) Clearly $\left.h^{\prime}\right|_{M \times S^{n-1}}=h$. Recall that $h=k_{1} \lambda_{1} g$ (Step 3) and 
$\lambda_{1}^{-1} \lg _{g\left(M_{0} \times B^{n}\right)}=$ identity. So for $x \in M_{0} \times B^{n}$,

$$
\begin{aligned}
h^{\prime}(x) & =g^{-1} k_{1}^{-1} \phi_{1}(k(x))=g^{-1}\left(k_{1}^{-1}(k(x))\right) \\
& =g^{-1} \lambda_{1}^{-1} k_{1}^{-1}(k(x))=\left(k_{1} \lambda_{1} g\right)^{-1}(k(x)) \\
& =k^{-1}(k(x))=x .
\end{aligned}
$$

Finally, for any $m \in M_{1}$, since $\phi_{1} k\left(\{m\} \times B^{n}\right) \subset \tilde{N}(a)$, where $k^{-1} \nu(a, 0) \in\{m\} \times$ $S^{n-1}$,

$$
\begin{aligned}
h^{\prime}\left(\{m\} \times B^{n}\right) & =g^{-1} k_{1}^{-1}\left(\phi_{1} k\left(\{m\} \times B^{n}\right)\right) \subset g^{-1} k_{1}^{-1}(\tilde{N}(a)) \\
& =g^{-1}\left(N^{\prime}(a)\right) \quad(\text { Step 3) } \\
& =g^{-1}\left(N_{1}\left(\lambda_{1}^{-1}(a)\right)\right) \quad(\text { Step 3) } \\
& =g^{-1}\left(N_{1}\left(a^{\prime}\right)\right) \quad(\text { Step 1) } \\
& =g^{-1}(O(m)) \quad(\text { Step 1) } \\
& =N(m) \times B^{n} .
\end{aligned}
$$

Thus $q h^{\prime}\left(\{m\} \times B^{n}\right) \subset N(m)$.

To finish the proof of Theorem 1, let us now consider the general case. Fix any $b_{0} \in S^{n-1}$. Define $H: M \times B^{n} \rightarrow M \times B^{n}$ by $H(m, b)=\left(h_{\#}^{-1}(m), b\right)$ where $h_{\#}: M$ $\rightarrow M$ is the homeomorphism induced by restricting $h$ on $M \times\left\{b_{0}\right\}$. Denote $N_{*}(m)$ $=h_{\#}^{-1}(N(m))$. Let $h_{*}=H h$. Then $\left.h_{*}\right|_{M \times\left\{b_{0}\right\}}=$ identity and for any $m$,

$$
q h_{*}\left(\{m\} \times B^{n}\right) \subset N_{*}(m) .
$$

In the subsequent argument of the special case, the factor $1 / k$ in defining $\varepsilon(m)$ is irrelevant except to provide a technical data needed in the proof of Theorem 2 . The important point is that $N(m)$ (hence $N_{*}(m)$ ) varies continuously with $m$. Thus there is a fibre homeomorphism $h_{*}^{\prime}: M \times B^{n} \rightarrow M \times B^{n}$ satisfying the conclusion of Theorem 1. Let $h^{\prime}=H^{-1} h_{*}$. $h^{\prime}$ is what we wanted.

\section{REFERENCES}

[An] R. D. Anderson, Spaces of homeomorphisms of finite graphs, Illinois J. Math. (to appear).

[An-Sc] R. D. Anderson and R. M. Schori, Factors of infinite-dimensional manifolds, Trans. Amer. Math. Soc. 142 (1969), 315-330.

[Ce] J. Cerf, Groupes d'áutomorphismes et groupes de difféomorphismes des variétés compactes de dimension 3, Bull. Soc. Math. France 87 (1959), 319-329.

[Ch] T. A. Chapman, Homeomorphisms of Hilbert-cube manifolds, Trans. Amer. Math. Soc. 152 (1973), 227-239.

[Ch-Wo, II] T. A. Chapman and R. Y. Wong, On homeomorphisms of infinite dimensional bundles. II, Trans. Amer. Math. Soc. 191 (1974), 261-268.

[Do] T. Dobrowolski, correspondence, 1982.

[Du] J. Dugundji, Topology, Allyn \& Bacon, Boston, Mass., 1966.

[Ed-Ki], R. D. Edwards and R. Kirby, Deformations of space of imbeddings, Ann. of Math. (2) 93 (1971), 63-88.

[Fe] S. Ferry, The homeomorphism group of a compact Hilbert cube manifold is an ANR, Ann. of Math. (2) 106 (1977), 101-119.

[Ge] R. Geoghegan, On spaces of homeomorphisms, embeddings and functions. I, Topology 11 (1972), 159-177.

[He] D. W. Henderson, Infinite-dimensional manifolds are open subsets of Hilbert space, Topology 9 (1970), 25-33. 
[Lu-Ma] R. Luke and W. K. Mason, The space of a compact two-manifold is an ANR, Trans. Amer. Math. Soc. 146 (1972), 275-285.

[Re] P. Renz', The contractibility of the homeomorphism group of some product spaces by Wong's method, Math. Scand. 28 (1971), 182-188.

$\left[\mathbf{T}_{\mathbf{0 1}}\right]$ H. Torunczyk, Absolute retracts as factors of normed linear spaces, Fund. Math. 86 (1974), 53-67.

$\left[\mathbf{T}_{\mathbf{0 2}}\right]$. Homeomorphism groups of compact Hilbert cube manifolds which are manifolds, Bulletin, Polish Academy of Science, 1977.

[We] J. E. West, Cartesian factors of infinite-dimensional spaces, Topology Conf. (VPI), Lecture Notes in Math., vol. 375, Springer, Berlin and New York, 1974.

[Wo] R. Y. Wong, On homeomorphisms of certain infinite dimensional spaces, Trans. Amer. Math. Soc. 128 (1967), 148-154.

Department of Mathematics, University of California at Santa Barbara, Santa Barbara, CALIFORNIA 93106 